\title{
A novel missense variant of the GNAI3 gene and recognisable morphological characteristics of the mandibula in ARCND1
}

\author{
Kumiko Yanagi ${ }^{1} \cdot$ Noriko Morimoto $^{2} \cdot$ Manami Iso $^{3} \cdot$ Yukimi Abe $^{1} \cdot$ Kohji Okamura $^{4}{ }^{4}$ Tomoo Nakamura ${ }^{5}$.

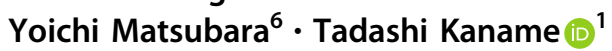

Received: 24 November 2020 / Revised: 9 February 2021 / Accepted: 21 February 2021 / Published online: 15 March 2021

(c) The Author(s) 2021. This article is published with open access

\begin{abstract}
Auriculocondylar syndrome (ARCND) is an autosomal monogenic disorder characterised by external ear abnormalities and micrognathia due to hypoplasia of the mandibular rami, condyle and coronoid process. Genetically, three subtypes of ARCND (ARCND1, ARCND2 and ARCND3) have been reported. To date, five pathogenic variants of GNAI3 have been reported in ARCND1 patients. Here, we report a novel variant of GNAI3 (NM_006496:c.807C>A:p.(Asn269Lys)) in a Japanese girl with micrognathia using trio-based whole exome sequencing analysis. The GNAI3 gene encodes a heterotrimeric guanine nucleotide-binding protein. The novel variant locates the guanine nucleotide-binding site, and the substitution was predicted to interfere with guanine nucleotide-binding by in silico structural analysis. Three-dimensional computer tomography scan, or cephalogram, displayed severely hypoplastic mandibular rami and fusion to the medial and lateral pterygoid plates, which have been recognised in other ARCND1 patients, but have not been described in ARCND2 and ARCND3, suggesting that these may be distinguishable features in ARCND1.
\end{abstract}

Supplementary information The online version contains supplementary material available at https://doi.org/10.1038/s10038021-00915-z.

Kumiko Yanagi

yanagi-kmk@ncchd.go.jp

$\triangle$ Tadashi Kaname

kaname-t@ncchd.go.jp

1 Department of Genome Medicine, National Center for Child Health and Development, Setagaya, Tokyo, Japan

2 Division of Otolaryngology, National Center for Child Health and Development, Setagaya, Tokyo, Japan

3 Department of Pharmacology, National Center for Child Health and Development, Setagaya, Tokyo, Japan

4 Department of Systems BioMedicine, National Center for Child Health and Development, Setagaya, Tokyo, Japan

5 Division of General Pediatrics \& Interdisciplinary Medicine, National Center for Child Health and Development, Setagaya, Tokyo, Japan

6 National Center for Child Health and Development, Setagaya, Tokyo, Japan

\section{Introduction}

Auriculocondylar syndrome (ARCND) is a rare autosomal dominant or recessive disorder characterised by recognisable malformation of the ears, known as question-mark ears, and micrognathia involving hypoplasia of the mandibular rami, condyle and coronoid process. These orofacial malformations lead to respiratory difficulties, including apnoea. Feeding and speech difficulties due to ankylosis of the temporomandibular joints often become the chief complaints [1].

ARCND is genetically classified into three subtypes, ARCND1 (MIM\#602483), ARCND2 (MIM \#614669) and ARCND3 (MIM \#615706), whose causative genes are GNAI3 [1-6], PLCB4 [2, 3, 7-9] and EDN1 [10], respectively. GNAI3 encodes guanine nucleotide-binding protein subunit $\alpha$ (Goi3), a member of the heterotrimeric guanine nucleotide-binding proteins ( $\mathrm{G}$ proteins). Goi3 (NP_006487.1) has five guanine nucleotide-binding sites, G1-G5 boxes, in the GTP catalytic domain. The amino acid sequences in the $\mathrm{G}$ boxes are essential to the binding of guanine nucleotides [11]. To date, five pathogenic variants have been reported: three variants in the G1 box (p.Gly40Arg, p.Gly45Val and p.Ser47Arg), one in the G4 box (p.Asn269Tyr) and one which locates just one amino acid outside of the G1 box (p.Thr48Asn) [2, 6, 10]. 
A

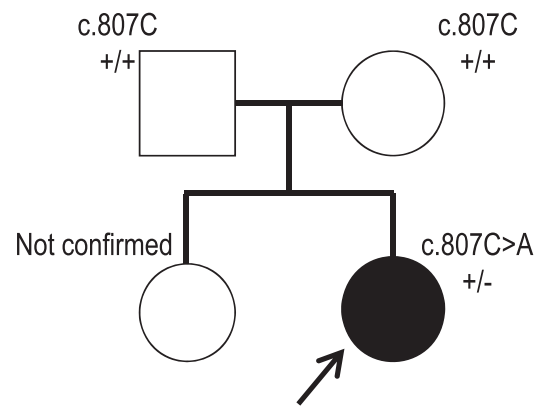

B

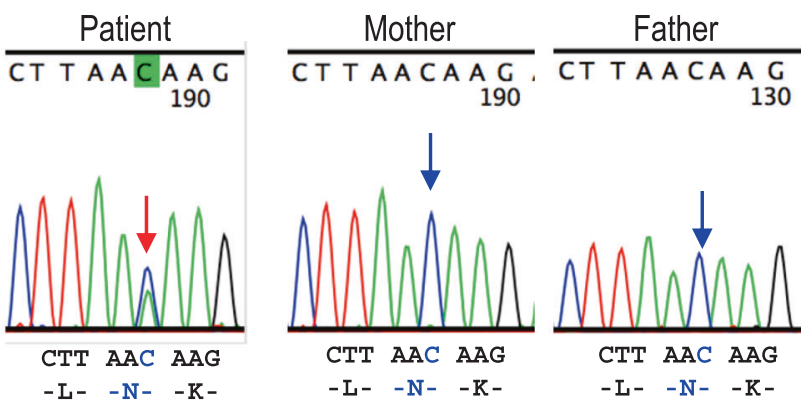

CTT AAA AAG

$-\mathrm{L}-\mathrm{K}-\mathrm{K}-$

C
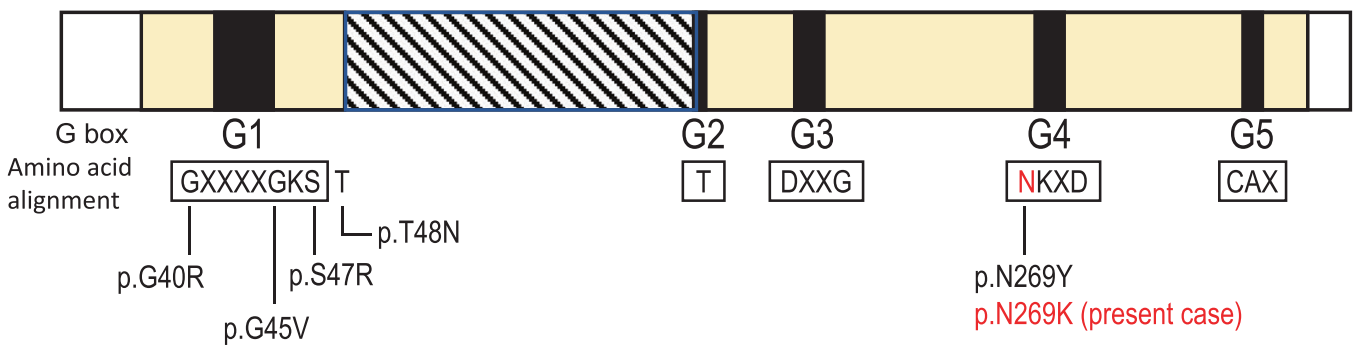

D
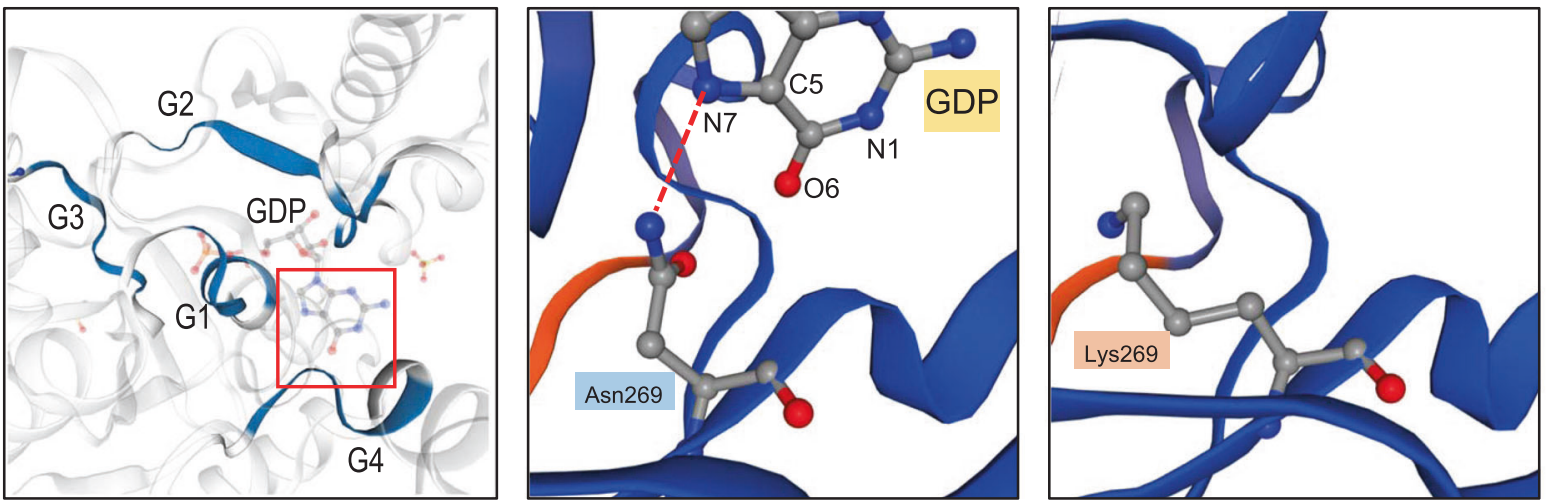

Fig. 1 Pedigree and genetic analysis. A Pedigree of the family. There was no family history. The proband has an elder sister with normal appearance. The mother has no history of trauma or exposure to any known teratogens or intake of any medication during pregnancy. B Electropherograms of Sanger sequence. The heterozygous de novo variant in the patient, c.807C >A (GNAI3:NM_006496), is indicated by the red arrow (left panel). The same nucleotide in her parents is indicated by the blue arrow (middle and right panel). The amino acids translated from the DNA sequences are presented under each electropherogram. C Schematic diagram of Goi3 (NP_006487.1). Five guanine nucleotide-binding site (G1-G5, black box) are located within GTP catalytic domains (yellow box). The catalytic domain is divided by an $\alpha$ helical domain (diagonal stripes). Consensus amino acid residues of the nucleotide-binding sites are boxed and indicated under each bonding site. Goi3 variants from the literature are indicated in black and the novel variant identified in this case is indicated in red. D In silico three-dimensional views of the Goi3. Wild-type Goi3 was obtained from a database of annotated 3D structures generated by SWISS-MODEL (UniProtKB AC; P08754, PDB ID; 4g5o). Regions of the guanine nucleotide-binding site, G1-G4, are indicated in blue. The GDP-binding region concerning Asn269 is indicated by the red square (left panel). The side chain of Asn269 in wild-type Goi3 makes a hydrogen bond to the N7 atom of the GDP (red broken line, middle panel). Lysin is a charged amino acid with a longer carbon skeleton compared to asparagine. The $\varepsilon$ amino group of Lys269 is facing the opposite side (arrow), which would affect the hydrogen bond to GDP (right panel, GDP is not shown). The structure of the Goi3 variant (p. Asn269Lys) was constructed based on the guanine nucleotide-binding protein $\mathrm{G}(\mathrm{k})$ subunit alpha by the SWISS-MODEL server homology modelling pipeline 
Here, we present a novel variant in the G4 box of Goi3 found in a Japanese girl tentatively diagnosed with severe micrognathia and describe recognisable morphological characteristics of the mandibula specific in ARCND1.

\section{Case report}

The proband was a 2-year-old Japanese girl born to healthy non-consanguineous parents (Fig. 1A). She presented severe mandibular hypoplasia and a rounded facial appearance with prominent cheeks. External ear malformation (question-mark ears) was observed. Oral malformations, such as microstomia, lobular hypoplastic tongue with soft-tissue projections, cleft palate with hypoplastic soft palate and ankylosis of the temporomandibular joints, were also present (Supplementary Figure). She also had difficulties with feeding and speech articulation. Meatal stenosis, malformation of the internal ear (semicircular and cochlear duct) and moderate sensorineural hearing loss were seen. Tracheostomy was performed due to upper airway obstruction. Three-dimensional computed tomography (3DCT) showed mandibular condyle agenesis, excessively short rami and retrognathia (Fig. 2). The mandibular rami were fused with medial and lateral pterygoid plates. Both her mandibular angles were unclear. The intracranial structure, nasal cavity, orbital cavity and maxilla were normal. No other general malformations, growth retardation or developmental delay was observed. The clinical findings for this case are summarised in Table 1.

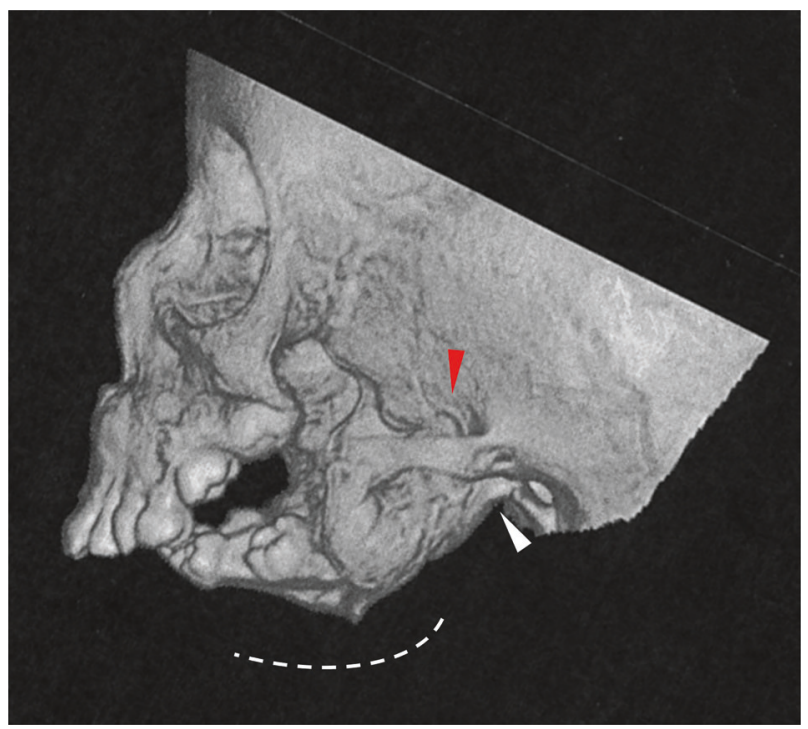

Fig. 2 Three-dimensional computed tomography (3D-CT) image of the patient. Mandibular condyle agenesis and excessively hypoplastic rami and processes can be observed. The possible positions of the mandibular jaw (white arrowhead) and process (red arrowhead) are indicated. The mandibular angle is unclear

\section{Genetic and protein structural analyses}

After obtaining written informed consent from her parents, whole exome sequencing (WES) analysis was performed in the patient and her parents (see Supplementary Methods) [12]. By trio-based filtering, we identified a novel de novo non-synonymous variant in exon 7 of GNAI3 (NM_006496.4:c.807C>A:p.(Asn269Lys)). The variant was confirmed by Sanger sequencing (Fig. 1B). It was not found in the ExAC, gnomAD, 1000 genome or the inhouse database of WES from over 4000 Japanese individuals. In silico prediction programmes, such as PolyPhen2 and SIFT, estimated that the GNAI3 novel variant was damaging. Asn 269 within the G4 box domain of the Goi3 forms a hydrogen bond with the N7 atom of the guanine moiety [13]. In silico structural analysis reveals that the substitution of Asn269 to Lys is expected to disrupt the hydrogen bond, which may interfere downstream of Goi3 signal (Fig. 1D). According to the ACMG guidelines, the novel variant was classified as likely pathogenic (strong, PS2; moderate, PM2 and PM5; supporting, PP2 and PP3).

\section{Discussion}

We report a patient with severe mandibular hypoplasia, external ear malformation and a rounded face with prominent cheeks. Trio-based WES analysis revealed a novel missense variant of Goi3, p.(Asn269Lys), which is predicted disruption of the hydrogen bond to the N7 atom of the guanine moiety. One patient with p.Asn269Tyr, which was different substitution to Tyr, was reported [6]. The N7 seems to be backbone of tryptophane, therefore hydrophobic interaction might be likely and easy disrupted by substitution of Lysine, supporting that mandibular hypoplasia phenotype in our patient (Supplementary Fig. 1) is more severe than in the patient with p.Asn269Tyr [6]. Her head 3D-CT showed agenesis of the mandibular condyle, retrognathia and an excessively short mandibular rami, which were fused with the medial and lateral pterygoid plates.

The orofacial appearance is similar among cases of ARCND1 (Table 1; for photographs, refer to original article) [1-6]. We investigated and compared the appearance of the mandibular area among cases of ARCND1 [2, 4, 5, 14], ARCND2 [2, 3, 7, 8] and ARCND3 [10] using 3D-CT images or radiological images. Anatomically, the distal region of the mandibula consists of the mandibular condyle, the coronoid process and the mandibular rami. The appearance in 3D-CT or radiological images in such mandibula are similar among ARCND1 cases. In our case, there was a lack of clarity of the mandibular angle due to the excess hypoplasia of the distal region and fusion between 


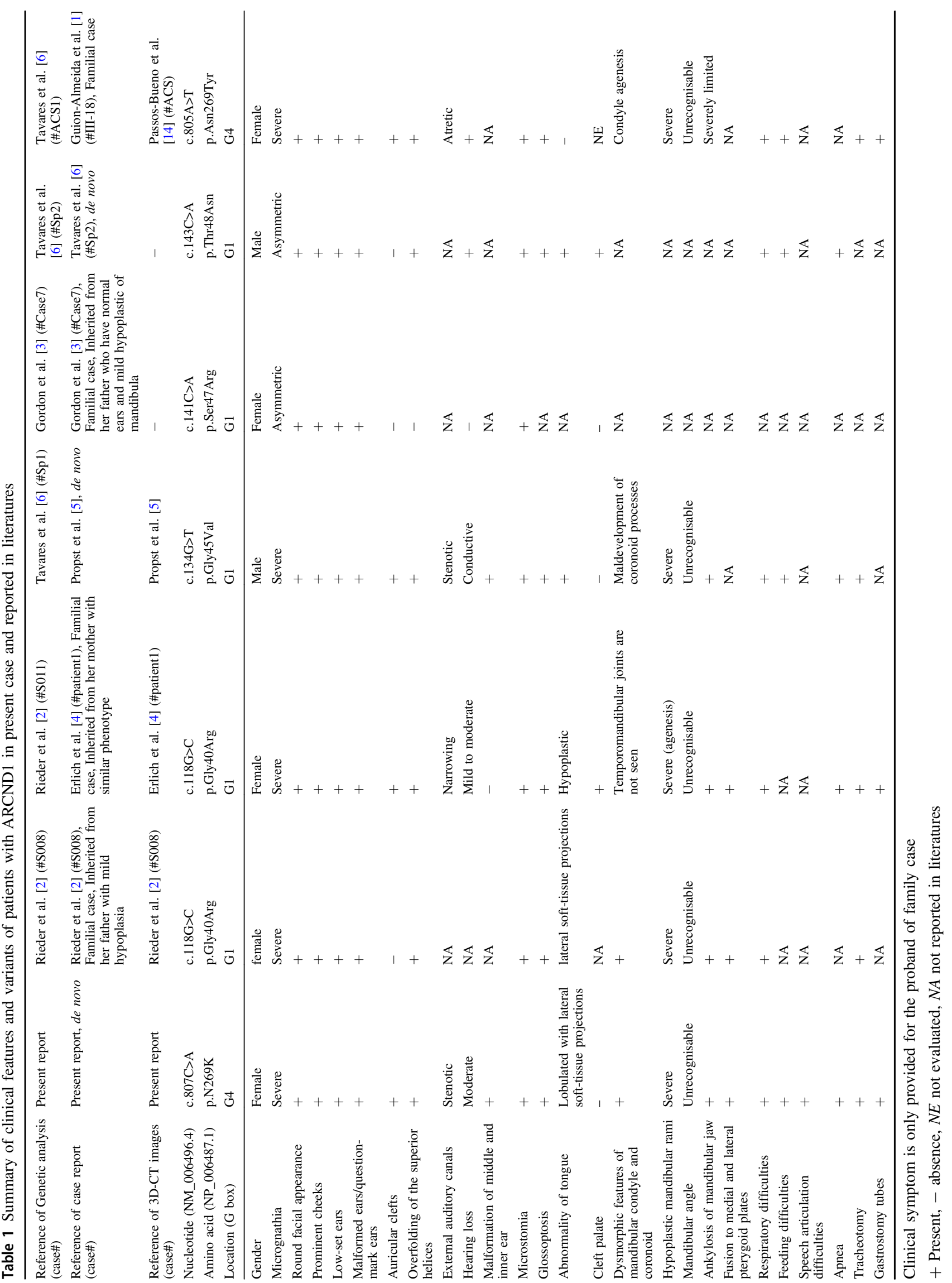


the mandibular rami and the pterygoid plate, which are common in ARCND1 patients (Table 1). In contrast, a clear mandibular angle and mild hypoplastic short mandibula are observed in patients with ARCND2 and ARCND3 (Supplementary Table S1). In addition, there is no description of fusion between the mandibular rami and the pterygoid plate in them (Supplementary Table S1). Although the obtained images from the literature were limited, these findings might be distinguishable features of ARCND1 from ARCND2 and ARCND3.

The development of the mandibula is uniquely regulated during embryogenesis and is also affected by postnatal food preference [15]. Dlx5/Dlx6 knockout mice show a hypoplastic mandibula similar to that found in ARCND [16]. Gnai3, Plcb4 and Edn1 act as upstream molecules in the endotherin-Dlx5/Dlx6 signalling pathway, and dysregulation of the pathway is involved in the pathogenicity of ARCND [2, 6, 17]. However, the mandibular hypoplasia of ARCND1 is much more severe than that of ARCND2 and ARCND3 (Table 1 and Supplementary Table S1). Another pathway may support such severity. It is interesting that both the mandibular condyle and the pterygoid process undergo cartilage and membrane ossification during their development and growth [18]. In mouse embryos, SRY-box 9 (Sox 9) was found to be first expressed in chondrocytes in the cranial base prior to expression of type $\mathrm{X}$ collagen at E14.5 and in chondrocytes of the condylar cartilage at E15.5 [18]. Both overexpression and knockout of Sox9 lead to dramatic inhibition of chondrocyte proliferation and terminal differentiation, which results in false endochondral-like ossification of these regions [19]. Sox9 is regulated by Gnai3 via PKA and cAMP [20]. Goi3 variants could also affect the SOX9 expression during embryogenesis, possibly causing the severity.

\section{Web resources}

Database. GnomAD: https://gnomad.broadinstitute.org. 1000 Genome. PolyPhen2: http://genetics.bwh.harvard.edu/ pph2/. SIFT: http://provean.jcvi.org/genome_submit_2. php?species=human. ACMG: https://www.nature.com/ gim/articles?type $=$ acmg-standards-and-guidelines. SWISSMODEL: https://swissmodel.expasy.org/interactive.

\section{Data availability}

The raw datasets are available from the corresponding author upon reasonable request.

Acknowledgements The authors would like to express our sincere thanks to the families, clinician and technical staff who took part in this presentation. We would like to thank Keiko Hayashi and Nana Kobayashi for whole exome sequence and management of samples. Our work described herein was aided by grants funding JSPS
KAKENHI (Scientific Research (C), 20 K09916 (KY) and Japan Agency for Medical Research and Development, 20ek0109301h (YM).

\section{Compliance with ethical standards}

Conflict of interest The authors declare no competing interests.

Ethical approval The patient and her family members gave written informed consent for genetic testing and the study was approved by the National Research Institute for Child Health and Development, Initiative on Rare and Undiagnosed Disease in Paediatrics (IRUD-P), Japan (https://www.amed.go.jp/en/index.html).

Publisher's note Springer Nature remains neutral with regard to jurisdictional claims in published maps and institutional affiliations.

Open Access This article is licensed under a Creative Commons Attribution 4.0 International License, which permits use, sharing, adaptation, distribution and reproduction in any medium or format, as long as you give appropriate credit to the original author(s) and the source, provide a link to the Creative Commons license, and indicate if changes were made. The images or other third party material in this article are included in the article's Creative Commons license, unless indicated otherwise in a credit line to the material. If material is not included in the article's Creative Commons license and your intended use is not permitted by statutory regulation or exceeds the permitted use, you will need to obtain permission directly from the copyright holder. To view a copy of this license, visit http://creativecommons. org/licenses/by/4.0/.

\section{References}

1. Guion-Almeida ML, Zechi-Ceide RM, Vendramini S, KokitsuNakata NM. Auriculo-condylar syndrome: additional patients. Am J Med Genet. 2002;112:209-14.

2. Rieder MJ, Green GE, Park SS, Stamper BD, Gordon CT, Johnson JM, et al. A human homeotic transformation resulting from mutations in PLCB4 and GNAI3 causes auriculocondylar syndrome. Am J Hum Genet. 2012;90:907-14.

3. Gordon CT, Vuillot A, Marlin S, Gerkes E, Henderson A, AlKindy A, et al. Heterogeneity of mutational mechanisms and modes of inheritance in auriculocondylar syndrome. J Med Genet. 2013;50:174-86.

4. Erlich MS, Cunningham ML, Hudgins L. Transmission of the dysgnathia complex from mother to daughter. Am J Med Genet. 2000;95:269-74.

5. Propst EJ, Ngan BY, Mount RJ, Martin-Munoz D, Blaser S, Harrison RV, et al. Ossicular fusion and cholesteatoma in auriculo-condylar syndrome: in vivo evidence of arrest of embryogenesis. Laryngoscope. 2013;123:528-32.

6. Tavares VLR, Gordon CT, Zechi-Ceide RM, Kokitsu-Nakata NM, Voisin N, Tan TY, et al. Novel variants in GNAI3 associated with auriculocondylar syndrome strengthen a common dominant negative effect. Eur J Hum Genet. 2015;23:481-5.

7. Leoni C, Gordon CT, Della Marca G, Giorgio V, Onesimo R, Perrino F, et al. Respiratory and gastrointestinal dysfunctions associated with auriculo-condylar syndrome and a homozygous PLCB4 loss-of-function mutation. Am J Med Genet A. 2016;170:1471-8.

8. Kokitsu-Nakata NM, Zechi-Ceide RM, Vendramini-Pittoli S, Romanelli Tavares VL, Passos-Bueno MR, Guion-Almeida ML. Auriculo-condylar syndrome. Confronting a diagnostic challenge. Am J Med Genet A. 2012;158A:59-65. 
9. Romanelli Tavares VL, Zechi-Ceide RM, Bertola DR, Gordon CT, Ferreira SG, Hsia GS, et al. Targeted molecular investigation in patients within the clinical spectrum of auriculocondylar syndrome. Am J Med Genet A. 2017;173:938-45.

10. Gordon CT, Petit F, Kroisel PM, Jakobsen L, Zechi-Ceide RM, Oufadem $\mathrm{M}$, et al. Mutations in endothelin 1 cause recessive auriculocondylar syndrome and dominant isolated question-mark ears. Am J Hum Genet. 2013;93:1118-25.

11. Neer EJ. G proteins: critical control points for transmembrane signals. Protein Sci. 1994;3:3-14.

12. Sasaki H, Yanagi K, Ugi S, Kobayashi K, Ohkubo K, Tajiri Y, et al. Definitive diagnosis of mandibular hypoplasia, deafness, progeroid features and lipodystrophy (MDPL) syndrome caused by a recurrent de novo mutation in the POLD1 gene. Endocr J. 2018;65:227-38.

13. Liu Shenping, Cerione RichardA, Clardy Jon. Structural basis for the guanine nucleotide-binding activity of tissue transglutaminase and its regulation of transamidation activity. PNAS. 2002;99:2743-7.

14. Passos-Bueno MR, Ornelas CC, Fanganiello RD. Syndromes of the first and second pharyngeal arches: a review. Am J Med Genet A. 2009;149A:1853-9.
15. Parada C, Chai Y. Mandible and tongue development. Curr Top Dev Biol. 2015;115:31-58.

16. Depew M, Simpson C, Morasso M, Rubenstein A. Reassessing the Dlx code: the genetic regulation of branchial arch skeletal pattern and development. J Anat. 2005;207:501-61.

17. Clouthier DE, Passos-Bueno MR, Tavares AL, Lyonnet S, Amiel J, Gordon CT. Understanding the basis of auriculocondylar syndrome: insights from human, mouse and zebrafish genetic studies. Am J Med Genet C Semin Med Genet. 2013; 163C:306-17.

18. Hirouchi H, Kitamura K, Yamamoto M, Odaka K, Matsunaga S, Sakiyama K, et al. Developmental characteristics of secondary cartilage in the mandibular condyle and sphenoid bone in mice. Arch Oral Biol. 2018;89:84-92.

19. Kim Y, Murao H, Yamamoto K, Deng JM, Behringer RR, Nakamura T, et al. Generation of transgenic mice for conditional overexpression of Sox9. J Bone Min Metab. 2011;29:123-9.

20. Huan W, Zhou X, Lefebvre V, Crombrugghe B. Phosphorylation of SOX9 by cyclic AMP-dependent protein kinase A enhances SOX9's ability to transactivate a Col2a1 chondrocyte-specific enhancer. Mol Cell Biol. 2000;20:4149-58. 